\title{
REFERENCES
}

1. Abraham J. Barshop, A miniature mathematical science, Brooklyn College Math. Mirror, Annual Bulletin of the Mathematics Club of Brooklyn College 7 (1939), 14-17.

2. Lawrence M. Graves, A finite Bolyai-Lobachevsky plane, Amer. Math. Monthly 69 (1962), 130-132.

3. L. Szamkolowicz, On the problem of existence of finite regular planes, Colloq. Math. 9 (1962), 245-250.

4. Bernard J. Topel, Bolyai-Lobachevski planes with finite lines, pp. 40-42, Notre Dame Univ. Press, Notre Dame, Ind., 1944 (Reports of a Mathematical Colloquium, Ser. 2, no. 5/6).

Oregon State University

\section{ON THE SECOND COHOMOLOGY GROUP OF A RAEHLER MANIFOLD OF POSITIVE CURVATURE}

R. L. BISHOP ${ }^{1}$ AND S. I. GOLDBERG ${ }^{2}$

1. Introduction. A. Andreotti and T. Frankel proved that a 4dimensional compact Kaehler manifold of strictly positive sectional curvature is analytically homeomorphic with complex projective 2space, and the latter conjectured that this is true in all dimensions [2], [5]. In this note, evidence is given in support of this conjecture. In fact, the following theorem is proved.

THEOREM 1. Let $M$ be a complete Kaehler manifold of strictly positive curvature. Then, the second Betti number $b_{2}(M, R)$ is $1, i . e ., \operatorname{dim} H^{2}(M, R)$ $=1$ where $H^{i}(M, R)$ is the ith cohomology group of $M$ with real coeffcients.

Since the 2 -form defined by the Ricci tensor of $M$ is closed, and in fact, is also co-closed if the scalar curvature is a constant, we obtain immediately

Corollary (Koszul-Matsushima [7], [9]). A homogeneous Kaehler manifold of strictly positive curvature is an Einstein space, i.e., a space of constant mean curvature.

Received by the editors August 10, 1963.

1 The research of this author was supported by the National Science Foundation.

2 The research of this author was supported by the Air Force Office of Scientific Research. 
The above theorem was established by M. Berger [1] for manifolds of dimension 4. We employ the same method of proof; however, his reduction of a 2-form of bidegree $(1,1)$ led to difficulties which could not be resolved in higher dimensions. For example, in dimension 6 , he proved that if the sectional curvatures are positive, and the ratio of the smallest to the largest sectional curvature is 0.15 , then $b_{2}(M, R)$ is 1 .

THEOREM 2. If holomorphic curvature is positive, i.e., $K(X, J X)>0$ for all $X$ (see §2), and the maximum holomorphic curvature is less than twice the minimum holomorphic curvature (i.e., $M$ is $\lambda$-holomorphically pinched with $\lambda>1 / 2)$, then $b_{2}(M, R)=1$.

This is an immediate consequence of the inequality

(see $[1$, formula $(4.5)]$ ).

$$
K(X, Y)+K(X, J Y) \geqq \frac{2 \lambda-1}{2}
$$

2. Notation and formulae. A Kaehler manifold is considered as a Riemannian manifold with metric $\langle$, $\rangle$ admitting a self-parallel skewsymmetric linear transformation field $J$ (the almost complex structure tensor) such that $J^{2}=-$ identity. The relationship between the curvature transformation $R(X, Y)\left(X, Y \in T_{P}\right.$ - the tangent space at $P \in M)$ and the metric is given by

$$
R(X, Y)=D_{[X, Y]}-\left[D_{X}, D_{Y}\right]
$$

where $D_{X}$ denotes the operation of covariant differentiation in the direction of $X$, and

$$
\begin{aligned}
2\left\langle X, D_{Z} Y\right\rangle= & Z\langle X, Y\rangle-X\langle Y, Z\rangle+Y\langle X, Z\rangle+\langle Z,[X, Y]\rangle \\
& -\langle X,[Y, Z]\rangle+\langle Y[X, Z]\rangle .
\end{aligned}
$$

Let $M$ be a Kaehler manifold with almost complex structure tensor $J$. Then, for any $X, Y \in T_{P}$,

(i) $R(J X, J Y)=R(X, Y)$,

(ii) $K(J X, J Y)=K(X, Y)$,

where $K(X, Y)$ is the sectional curvature function, and when $X, Y$, $J X, J Y$ are orthonormal,

(iii) $\langle R(X, J X) Y, J Y\rangle=K(X, Y)+K(X, J Y)$.

3. Normalization of a real form of bidegree $(1,1)$. The idea of the proof of Theorem 1 is to show that a real harmonic 2 -form $\alpha$ has the property that at each point $P \in M$ there is an orthonormal basis $\left\{X_{i}, J X_{i}\right\}$ of $T_{P}, i=1, \cdots, \operatorname{dim}_{C} M$, such that the only nonzero 
components of $\alpha$ are $\alpha_{i i^{*}}=\alpha\left(X_{i}, J X_{i}\right), i^{*}=\operatorname{dim}_{C} M+1, \cdots, \operatorname{dim} M$. It will then follow that $\alpha$ is a multiple of the fundamental form $\Omega$ of $M$. In this connection Berger [1] proved the following result:

If a real harmonic 2-form $\alpha$ of bidegree $(1,1)$ on a compact Kaehler manifold $M$ of strictly positive curvature has the property that at each point $P \in M$ there is an orthonormal basis $\left\{X_{i}, J X_{i}\right\}$ of $T_{P}$ such that the only nonvanishing components of $\alpha$ are $\alpha\left(X_{i}, J X_{i}\right)$ then $\alpha$ is a multiple of the Kaehler form $\Omega$ of $M$. If the curvature requirement is replaced by " $M$ is holomorphically pinched with holomorphic pinching $>1 / 2$, , the same conclusion prevails.

We show that every harmonic 2 -form of bidegree $(1,1)$ has the required normalization. In fact, it is shown that every real form of bidegree $(1,1)$ can be so normalized.

Lемма. Let $\xi$ be a real form of bidegree $(1,1)$. Then, there is an orthonormal basis $\left\{X_{i}, J X_{i}\right\}$ such that the only nonzero components of $\xi$ are of the form $\xi\left(X_{i}, J X_{i}\right)$.

Proof. Let $S(X, Y)=\xi(J X, Y)$. The fact that $\xi$ is of bidegree $(1,1)$ is equivalent to $\xi(X, Y)=\xi(J X, J Y)$ for any $X$ and $Y$. Thus, for $S$ we have

$$
S(X, Y)=-\xi(X, J Y)=\xi(J Y, X)=S(Y, X) .
$$

Moreover,

$$
S(J X, J Y)=-\xi(X, J Y)=S(X, Y) .
$$

Hence, $S$ is a symmetric bilinear form which is invariant under $J$. If $X_{1}$ is a characteristic vector of $S$, so is $J X_{1}$. Consequently, we can choose an orthonormal basis $\left\{X_{i}, J X_{i}\right\}$ inductively so that the only nonvanishing components of $S$ are of the form $S\left(X_{i}, X_{i}\right)$ $=S\left(J X_{i}, J X_{i}\right)$, which reverts to the desired statement for $\xi$.

4. Proof of Theorem 1. Since curvature is positive, there are no holomorphic 2-forms [3]. Consequently, a harmonic 2-form is of bidegree $(1,1)$. The remainder of the proof is standard, i.e., the Bochner-Lichnerowicz technique is applied [4], [6], [8]. Indeed, the essential step is to make proper use of the formula

where

$$
F(\xi)=\sum_{i} \sum_{j \neq i, i^{*}}\left(K_{i j}+K_{i j^{*}}\right)\left(\xi_{i i^{*}}\right)^{2}-4 \sum_{i<j} R_{i i^{*} j j^{*}} \xi_{i i^{*}} \xi_{j j^{*}}
$$

$$
K_{i j}=K\left(X_{i}, X_{j}\right), K_{i j^{*}}=K\left(X_{i}, J X_{j}\right) \text { and } R_{i i^{*} j j^{*}}=\left\langle R\left(X_{i}, J X_{i}\right) X_{j}, J X_{j}\right\rangle \text {. }
$$

In fact, this is what Berger did to obtain his result quoted above, from which Theorems 1 and 2 follow by use of the lemma. 
Added in proof. Theorem 2 is a substantial improvement of a corresponding result due to $\mathrm{S}$. Kobayashi [Topology of positively pinched Kaehler manifolds, Tôhoku Math. J. 15 (1963), 121-139] who showed that if $\lambda>(22 m-17) /(26 m-19)$, where $m=\operatorname{dim}_{c} M$, then $b_{2}(M, R)$ $=1$.

Incidentally, the proof of the main result in his paper is greatly simplified by virtue of Theorem 1 above (see also [R. L. Bishop and S. I. Goldberg, On the topology of positively curved Kaehler manifolds, Tôhoku Math. J: 15 (1963), 359-364]).

\section{REFERENCES}

1. M. Berger, Pincement riemannien et pincement holomorphe, Ann. Scuola Norm. Sup. Pisa (3) 14 (1960), 151-159.

2. R. L. Bishop and S. I. Goldberg, On curvature and Euler-Poincare characteristic, Proc. Nat. Acad. Sci. U.S.A. 49 (1963), 814-817.

3. S. Bochner, Vector fields and Ricci curvature, Bull. Amer. Math. Soc. 52 (1946), 776-797.

4. - Curvature and Betti numbers, Ann. of Math. (2) 49 (1948), 379-390.

5. T. Frankel, Manifolds with positive curvature, Pacific J. Math. 11 (1961), $165-174$.

6. S. I. Goldberg, Curvature and homology, Academic Press, New York, 1962.

7. J.-L. Koszul, Sur la forme hermitienne canonique des espaces homogènes complexes, Canad. J. Math. 7 (1955), 562-576.

8. A. Lichnerowicz, Courbures, nombre de Betti et espaces symetriques, Proc. Internat. Congress of Mathematicians, Canibridge, Mass., 1950, Vol. 2, pp. 216223, Amer. Math. Soc., Providence, R. I., 1952.

9. Y. Matsushima, Sur les espaces homogènes kähleriens d'un groupe de Lie reductif, Nagoya Math. J. 11 (1957), 53-60.

UNIVERSITY OF ILLINOIS 